\title{
Identification of tRNA-derived fragments in colon cancer by comprehensive small RNA sequencing
}

\author{
WEI XIONG $^{1 *}$, XIAOLI WANG ${ }^{1 *}$, XINYI CAI $^{2^{*}}$, WEI XIONG ${ }^{2}$, YAN LIU $^{1}$, \\ CHENG LI ${ }^{1}$, QIUYAN LIU ${ }^{1}$, JIYONG QIN ${ }^{1}$ and YUNFENG LI $^{2}$ \\ Departments of ${ }^{1}$ Radiation Oncology and ${ }^{2}$ Colorectal Surgery, Yunnan Cancer Hospital, \\ The Third Affiliated Hospital of Kunming Medical University, \\ Kunming, Yunnan 650100, P.R. China
}

Received April 24, 2018; Accepted November 29, 2018

DOI: $10.3892 /$ or.2019.7178

\begin{abstract}
Transfer RNA-derived fragments (tRFs) are a novel class of small non-coding RNAs that are abundant in various species and have been implicated in human diseases. However, to the best of our knowledge, the role of tRFs in colon cancer has not been explored. In the present study, a comprehensive analysis was conducted to identify the tRFs associated with colon cancer and predict their roles in colon carcinogenesis. tRFs and miRNAs that were differentially expressed (DE) between colon cancer and matched peritumor tissues were identified by small RNA sequencing. DE mRNAs were screened by Gene Expression Profiling Interactive Analysis. DE microRNAs (miRNAs) reported to be associated with colon cancer were selected as key miRNAs. The targets of the DE tRFs and key miRNAs were predicted using MiRanda and RNAhybrid, respectively. The potential targets that were common to and negatively correlated with the DE tRFs and key miRNAs were screened. The DE mRNAs and potential targets were subjected to Gene Ontology and pathway analyses, respectively. In total, 16 DE tRFs, 26 DE miRNAs, and 5,327 DE mRNAs were identified. Five of the $26 \mathrm{DE}$ miRNAs were selected as key miRNAs. DE mRNAs were mainly enriched in cell proliferation-related processes and pathways. Fifty-five DE mRNAs
\end{abstract}

Correspondence to: Mr. Yunfeng Li, Department of Colorectal Surgery, Yunnan Cancer Hospital, The Third Affiliated Hospital of Kunming Medical University, 519 Kunzhou Road, Xishan, Kunming, Yunnan 650100, P.R. China

E-mail: liyunfengt@163.com

Mr. Jiyong Qin, Department of Radiation Oncology, Yunnan Cancer Hospital, The Third Affiliated Hospital of Kunming Medical University, 519 Kunzhou Road, Xishan, Kunming, Yunnan 650100, P.R. China

E-mail: qinjiyongt@163.com

*Contributed equally

Key words: colon cancer, small RNA sequencing, transfer RNA-derived fragments, tumorigenesis were potential targets of both DE tRFs and key miRNAs and were primarily enriched in vitamin metabolic pathways and the cyclic guanine monophosphate-protein kinase $\mathrm{G}$ signaling pathway. Collectively, these results suggest that tRFs may play crucial roles in the development of colon cancer. This study could provide valuable cues for further research into the roles of tRFs in colon cancer.

\section{Introduction}

Colon cancer is the most commonly diagnosed cancer and one of the deadliest diseases worldwide (1). Despite extensive research into strategies to treat colon cancer, conventional approaches have shown little promise in improving prognosis (2). The overall survival rate of patients who receive chemotherapy is only $10 \%$ (3). Thus, it is crucial to study the mechanism of colon carcinogenesis and to establish a theoretical basis for its treatment.

TransferRNA(tRNA)-derived fragments(tRFs), a novel type of small non-coding RNA that originates from tRNAs (4-6), are associated with several cellular activities (7-9). Previously, small non-coding RNAs, including microRNAs (miRNAs), tRFs, and piwi-interacting RNAs (piRNAs), have been implicated in tumorigenesis $(10,11)$. Among the tRFs, tRF-544 and tRF-315 are potential prognostic biomarkers for prostate cancer (12). tRF-1001 is expressed highly in many proliferating tumor cells and positively regulates cell proliferation (6). High expression of the tRFs ts-46 and ts-47 in lung cancer cell lines was identified to suppress cell proliferation (13). tRFs have been isolated as part of Argonaute (AGO) complexes, which implies that tRFs play a functional role, similar to that of miRNAs, and possess the ability to suppress mRNA translation in human B cells (14). An association with AGO implies that tRFs may be regulators of mRNA expression. miR-2476, which is one base pair different from the 5'-tRF derived from tRNA-Glu-CTC, is now not available due to its location near a known tRNA (15). miR-3676, which is in fact a tRF, targets the $3^{\prime}$ untranslated region (3'UTR) of the oncogene TCL1 and downregulates the expression of TCL1 $(13,16)$. Collectively, these data suggest that tRFs play key roles in various cancers and may be involved in tumorigenesis via post-translational alteration of gene expression. However, the role of tRFs in 
colon cancer remains unclear. Therefore, a comprehensive analysis was conducted to explore the role of tRFs in colon cancer.

In the present study, tRFs that were differentially expressed (DE) between colon cancer and normal peritumor tissues were identified by small RNA sequencing. The target genes of DE tRFs and known colon cancer-associated miRNAs were predicted to study the functional roles of tRFs in colon cancer by integrative analysis of tRFs, mRNAs and miRNAs.

\section{Materials and methods}

Sample collection. Tumor and matched normal peritumor tissues were collected from surgical specimens obtained from patients who underwent surveillance colonoscopy or routine screening in Department of Radiation Oncology, The Third Affiliated Hospital of Kunming Medical University (Kunming, China) between July 2016 and January 2018. A total of 30 patients who had not received any drug treatment before surgery were included. Three pairs of samples were used for small RNA sequencing and all 30 pairs of samples were used for reverse transcription-quantitative polymerase chain reaction (RT-qPCR) validation. All tissues were preserved in liquid nitrogen immediately after resection. The present study was approved by the Human Ethics Committee of Kunming Medical University. Written informed consent was obtained from all patients. Subjects with familial cancers, including familial adenomatous polyposis and Lynch syndrome, history of prior colonic resections or inflammatory bowel syndrome were excluded. The demographics and clinical characteristics of patient samples with low or moderate/high differentiation level according to histological characteristics (17) are presented in Table I. Characteristics recorded include sex, age, tumor size, location of hemicolon, TNM stage (18), lymph node and distant metastases.

RNA isolation, construction of small RNA library and sequencing. Total RNA was isolated from colon cancer and matched peritumor tissues with TRIzol reagent (Invitrogen; Thermo Fisher Scientific, Inc., Waltham, MA, USA). The quantity and purity of the total RNA were analyzed using NanoDrop (Thermo Fisher Scientific, Inc.) and $1 \%$ agarose gel electrophoresis. Purified and quantified RNA was used to construct small RNA libraries using the NEBNext Multiplex Small RNA Library Prep kit for Illumina (New England Biolabs, Inc., Ipswich, MA, USA) according to the manufacturer's protocol. Finally, small RNA sequencing was performed on HiSeq 2500 (Illumina, Inc., San Diego, CA, USA).

Data filtering and mapping. The raw sequencing data were refined with FastQC, which included filtering out low-quality and short reads $(<15$ nucleotides). The clean small RNA reads were mapped using the miRBase database (http://www. mirbase.org/) to identify known miRNAs, and the unmapped reads were further aligned with sequences in the Genomic tRNA Database (GtRNAdb, http://gtrnadb.ucsc.edu/). The reads mapped to GtRNAdb sequences were then mapped to those in tRFdb (http://genome.bioch.virginia.edu/trfdb/) and MINTbase (https://cm.jefferson.edu/MINTbase/) to identify tRFs. Unmapped reads from the GtRNAdb analysis were aligned to the piRNA sequences from the National Center for Biotechnology Information (https://www.ncbi.nlm.nih. gov/) and small nucleolar RNA (snoRNA) and small nuclear RNA (snRNA) sequences from Rfam (http://rfam.xfam. org/) $(19,20)$.

Screening of DE tRFs, miRNAs, and mRNAs. DE tRFs and miRNAs between the colon cancer and normal tissues were screened using the respective EBseq packages (http://www. bioconductor.org/packages/devel/bioc/html/EBSeq.html). The cut-off thresholds were set to $\mid \log _{2}$ (fold change) $\mid>1$, and false-discovery rate (FDR) was set to $<0.05$. Gene Expression Profiling Interactive Analysis (GEPIA; http://gepia.cancer-pku. cn/index.html) (21), an interactive website based on the expression profiles of tumor and normal samples from The Cancer Genome Atlas and the Genotype-Tissue Expression projects, provides tools for gene functional analysis, including tumor/normal differential expression analysis according to cancer types or pathological stages, correlation analysis, similar gene detection and patient survival analysis. DE mRNAs between colon adenocarcinoma and paired normal samples were identified using limma on GEPIA, with the following parameters: $\log _{2}$ (fold change) $\mid>1$, FDR $<0.05$.

Prediction of target genes of DE tRFs and key miRNAs. DE miRNAs implicated in colon cancer were selected as key miRNAs.MiRanda (http://www.microrna.org/microrna/home. do) and RNAhybrid (https://bibiserv.cebitec.uni-bielefeld. de/rnahybrid/) were used to predict the binding of DE tRFs or key miRNAs to the putative target genes. Genes with overlapping MiRanda (score $\geq 150$ and energy $\leq 20$ ) and RNAhybrid (energy $\leq 25$ ) results were considered as potential targets of tRFs or miRNAs. Next, the potential target genes common to tRFs and miRNAs were compared with DE mRNAs between colon adenocarcinoma and paired normal samples. Only the target genes that showed significant DE between the colon cancer and normal tissues and correlated negatively with the DE tRFs and candidate miRNAs were screened for further analysis. An integrated tRF/mRNA/miRNA network was then constructed using Cytoscape software (version 3.5.1) (22).

Gene Ontology $(G O)$ and pathway analyses of predicted target genes. GO (http://www.geneontology.org/) analysis (23) was performed to analyze the functions of the screened target genes $(24,25)$. Pathway enrichment analysis of the screened target genes was performed using the Kyoto Encyclopedia of Genes and Genomes (KEGG; http://www.kegg.jp/) (23). The cut-off threshold was set to $\mathrm{P}<0.05$.

$R T-q P C R$. Total RNAs from colon cancer tissues and normal peritumor tissue, colon cell lines and the human colon epithelial cell line were extracted with TRIzol reagent (Invitrogen; Thermo Fisher Scientific, Inc.) and reverse transcribed into cDNA using a RevertAidAM First Strand cDNA Synthesis kit (\#K1622; Thermo Fisher Scientific, Inc.) according to the manufacturer's protocol. qPCR was performed on the ABI Q6 detection system (Applied Biosystems; Thermo Fisher Scientific, Inc.) using a Real Time SYBR Master Mix kit (cat. no. 33041; Qiagen, Inc., Valencia, CA, USA). The thermocycling conditions were: $95^{\circ} \mathrm{C}$ for $10 \mathrm{~min}$ for 1 cycle; 
Table I. Demographics and clinical characteristics of patients with low $(n=10)$ or moderate/high $(n=20)$ differentiation.

\begin{tabular}{|c|c|c|c|c|}
\hline Characteristic & $\mathrm{n}$ & Low differentiation, $\mathrm{n}(\%)$ & Moderate/high differentiation, $\mathrm{n}(\%)$ & P-value \\
\hline Sex & & & & 0.698 \\
\hline Male & 15 & $5(50)$ & $10(50)$ & \\
\hline Female & 15 & $5(50)$ & $10(50)$ & \\
\hline Age, years & & & & 0.896 \\
\hline$>60$ & 17 & $6(60)$ & $11(55)$ & \\
\hline$\leq 60$ & 13 & $4(40)$ & $9(45)$ & \\
\hline Tumor size, $\mathrm{cm}$ & & & & 0.897 \\
\hline$>12$ & 16 & $6(60)$ & $10(50)$ & \\
\hline$\leq 12$ & 14 & $4(40)$ & $10(50)$ & \\
\hline Location of hemicolon & & & & 0.896 \\
\hline Left & 17 & $6(60)$ & $11(55)$ & \\
\hline Right & 13 & $4(40)$ & $9(45)$ & \\
\hline TNM stage & & & & 0.897 \\
\hline $\mathrm{I}+\mathrm{II}$ & 14 & $4(40)$ & $10(50)$ & \\
\hline III+IV & 16 & $6(60)$ & $10(50)$ & \\
\hline Lymph node metastasis & & & & 0.897 \\
\hline Yes & 14 & $5(50)$ & $9(45)$ & \\
\hline No & 16 & $5(50)$ & $11(55)$ & \\
\hline Distant metastasis & & & & 0.519 \\
\hline Yes & 3 & $2(20)$ & $1(5)$ & \\
\hline No & 27 & $8(80)$ & $19(95)$ & \\
\hline
\end{tabular}

TNM, tumor-node-metastasis.

Table II. Primer sequences for quantitative polymerase chain reaction.

Primer

\section{U6-F}

U6-R

hsa-miR-378a-3pF

hsa-miR-424-3pF

hsa-miR-483-3pF

hsa-miR-615-3pF

tRF-24-NMEH623K25F

tRF-30-XSXMSL73VL4YF

tRF-29-QU7BPN6ISBJOF

tRF-27-Q99P9P9NH5NF

$\mathrm{miR} / \mathrm{tRF}-\mathrm{R}$
Sequence $\left(5^{\prime}-3^{\prime}\right)$

CGATACAGAGAAGATTAGCATGGC

AACGCTTCACGAATTTGCGT

CGCAGACTGGACTTGGAGTCA

GCTGCAAAACGTGAGGCG

GGGCAAATCACTCCTCTCCTC

GCAGTCCGAGCCTGGGTCT

CAGCTGTCACGCGGGAGA

TGCCGTGATCGTATAGTGGTTAG

GCTGAGTGAAGCATTGGACTGTAA

GCAGGCTTCTGTAGTGTAGTGGTTA

AGTGCGTGTCGTGGAGTCG

All the miRs/tRFs shared the same reverse primer, miR/tRF-R. miR, microRNA; tRF, transfer RNA-derived fragment.

$95^{\circ} \mathrm{C}$ for $15 \mathrm{sec}$ and $60^{\circ} \mathrm{C}$ for $60 \mathrm{sec}$ for 45 cycles. Relative expression was calculated using the comparative threshold $(\mathrm{Ct})$ cycle method $\left(2^{-\Delta \Delta \mathrm{Cq}}\right)(26)$. U6 was used as an internal reference for the relative DE miRNA and tRF expression. Primers (Yingbiotech Co., Shanghai, China) and sequences are presented in Table II.
Cell culture. Human colon cancer cell lines SW480, SW620, HCT116, HT29 and human colon epithelial cell line NCM460 were purchased from the Type Culture Collection of the Chinese Academy of Sciences (Shanghai, China). HCT116 and SW480 cells were maintained by serial passage in RPMI-1640 medium (Corning, Inc., Corning, NY, USA) supplemented 


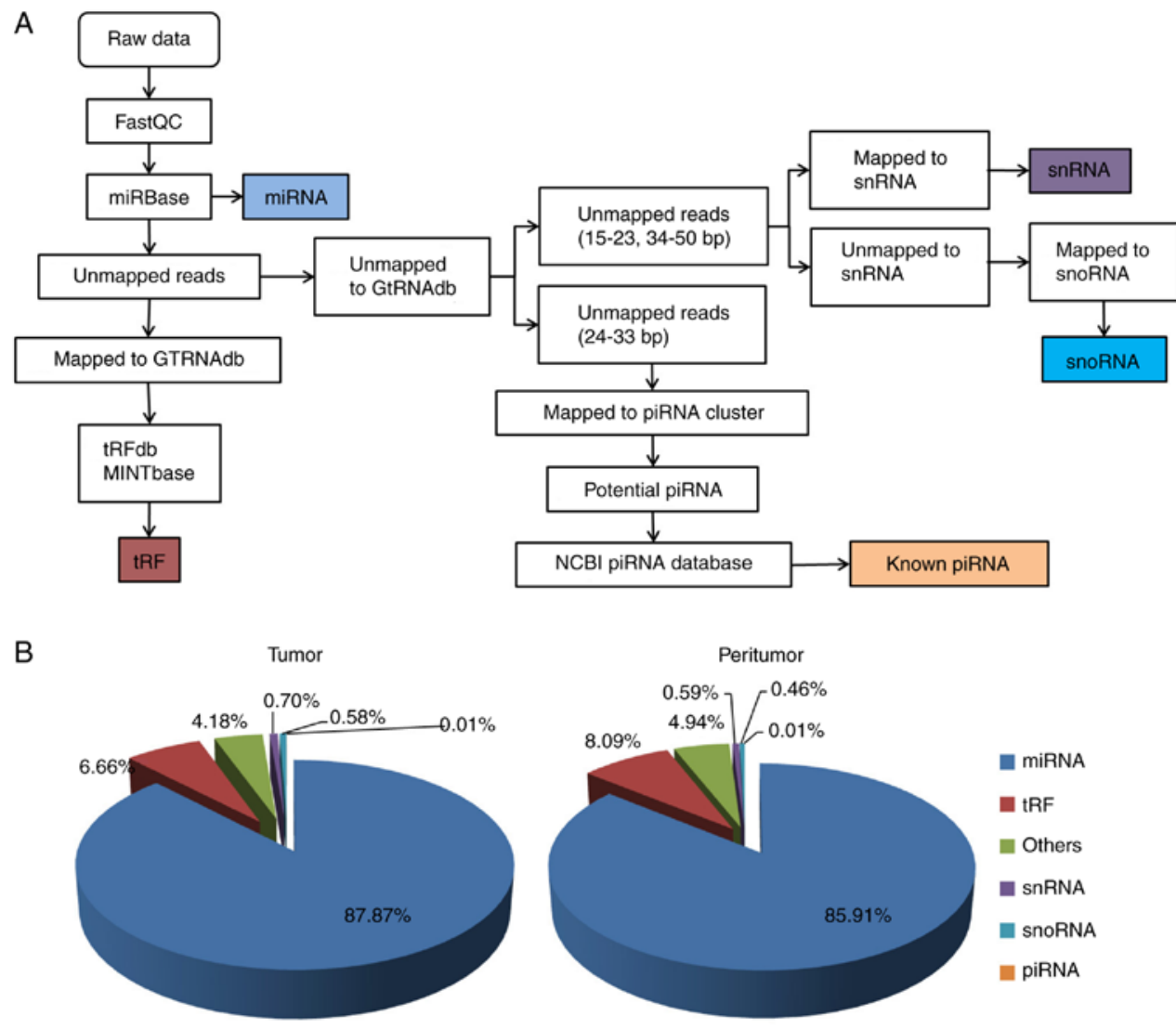

Figure 1. Flow chart and results of sequencing read mapping. (A) Flow chart of the sequencing read mapping process. (B) Types of small non-coding RNAs detected in colon cancer tissues and peritumor tissues. miRNA, microRNA; tRF, transfer RNA-derived fragment; snRNA, small nuclear RNA; snoRNA, small nucleolar RNA; piRNA, piwi-interacting RNA.

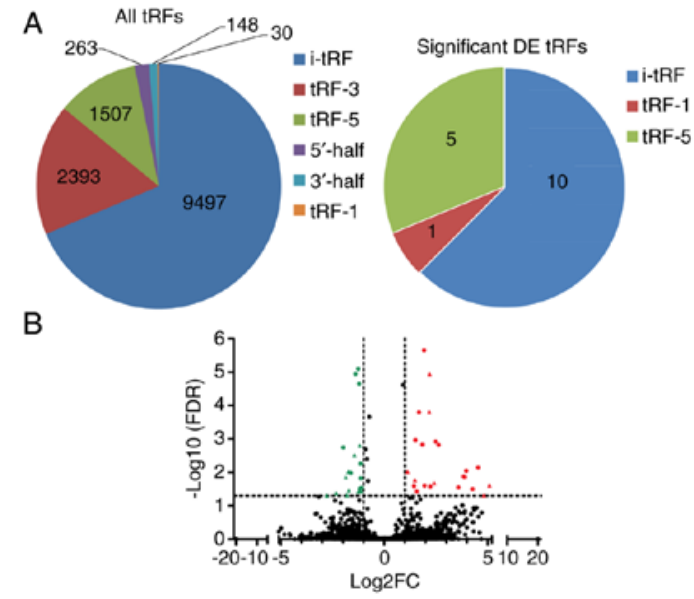

Figure 2. Detected tRFs in colon cancer and peritumor tissues. (A) All tRFs detected (left) and significant DE tRFs (right). (B) Volcano plot of DE tRFs and miRNAs between colon cancer and peritumor tissues. Triangles and dots indicate DE tRFs and miRNAs, respectively. Green and red indicate significantly downregulated and upregulated genes, respectively. miRNA microRNA; tRF, transfer RNA-derived fragment; DE, differentially expressed; FC, fold change; FDR, false discovery rate.

with $10 \%$ fetal bovine serum (FBS; Thermo Fisher Scientific, Inc.), $10 \mathrm{U} / \mathrm{ml}$ penicillin and $10 \mu \mathrm{g} / \mathrm{ml}$ streptomycin $(\mathrm{P} / \mathrm{S}$; Thermo Fisher Scientific, Inc.), and cultured in a $5 \% \mathrm{CO}_{2}$, humidified atmosphere at $37^{\circ} \mathrm{C}$. HT29 cells were maintained in McCoy's 5A medium (Thermo Fisher Scientific, Inc.) with
Table III. Numbers of detected small non-coding RNAs.

\begin{tabular}{lcc}
\hline Type & Total & Differentially expressed \\
\hline miRNA & 2,588 & 26 \\
tRF & 13,838 & 16 \\
piRNA & 167 & 0 \\
\hline
\end{tabular}

miRNA, microRNA; tRF, transfer RNA-derived fragment; piRNA, piwi-interacting RNA.

$10 \% \mathrm{FBS}$ and $1 \% \mathrm{P} / \mathrm{S}$ and cultured in a $5 \% \mathrm{CO}_{2}$, humidified atmosphere at $37^{\circ} \mathrm{C}$. SW620 cells were cultured in $\mathrm{L} 15$ medium (Beijing Solarbio Science \& Technology Co., Ltd., Beijing, China) with $10 \% \mathrm{FBS}$ and $1 \% \mathrm{P} / \mathrm{S}$ and cultured in $100 \%$ air at $37^{\circ} \mathrm{C}$. Dulbecco's modified Eagle's medium (Thermo Fisher Scientific, Inc.) and F12 medium (Thermo Fisher Scientific, Inc.) were used to culture the human colon epithelial cell line NCM460 with $10 \% \mathrm{FBS}$ and $1 \% \mathrm{P} / \mathrm{S}$, in a $5 \% \mathrm{CO}_{2}$, humidified atmosphere at $37^{\circ} \mathrm{C}$.

Statistical analysis. Statistical analysis was performed on SPSS version 12.0 statistical software (SPSS, Inc., Chicago, IL, USA) and the data were presented using the software GraphPad Prism 5.0 (GraphPad Software, Inc., La Jolla, CA, USA). Associations between demographics and differentia- 
Table IV. Significantly differentially expressed tRFs between colon cancer tissues and peritumor tissues.

\begin{tabular}{lcclcc}
\hline Database ID & Log $_{2}$ FC & FDR & \multicolumn{1}{c}{ Fragment sequence } & Type & Anticodon \\
\hline tRF-28-6Y1UFQ9D9ODD & 4.82 & 0.048029 & GGCTGAGTGAAGCATTGGACTGTAAATC & i-tRF & TyrGTA \\
tRF-25-6YYDLBRY73 & -2.78 & 0.048997 & GGCTGTTAACCGAAAGGTTGGTGGT & i-tRF & AsnGTT \\
tRF-16-9L5HMVE & -1.18 & 0.001581 & TGGTAGAGCGCGTGCT & i-tRF & AlaCGC, \\
& & & & & AlaAGC \\
tRF-22-9LON4VN11 & -1.26 & 0.038332 & TGGTAGAATTCTCGCCTGCCAC & i-tRF & GlyGCC \\
tRF-25-P940KK5Y93 & -1.44 & 0.003051 & GCCTCCTAAGCCAGGGATTGTGGGT & i-tRF & ArgCCT \\
tRF-24-PY8HM2OSIZ & -1.71 & 0.033956 & GCCTGTCACGCGGGAGACCGGGGT & i-tRF & AspGTC \\
tRF-38-YDLBRY73W0 & -1.80 & 0.047935 & TTAACCGAAAGGTTGGTGGTTCGA & i-tRF & AsnGTT \\
K5KKDR & & & GCCCACCCAGGGAC & & \\
tRF-29-P27JPJ60MVJY & -2.33 & 0.041736 & GCAGAGTGGCGCAGCGGAAGCGTGCTGGG & i-tRF & MetCAT \\
tRF-24-NMEH623K25 & -1.73 & 0.009462 & CTGTCACGCGGGAGACCGGGGTTC & i-tRF & AspGTC \\
tRF-29-QU7BPN6ISBJO & 5.08 & 0.024387 & GCTGAGTGAAGCATTGGACTGTAAATCTA & i-tRF & TyrGTA \\
tsRNA-1020 & -1.87 & 0.014078 & GAGAGCGCTCGGTTTTT & tRF-1 & PheGAA \\
tRF-27-Q99P9P9NH5N & 2.17 & $1.53 \times 10^{-4}$ & GCTTCTGTAGTGTAGTGGTTATCACGT & tRF-5 & ValCAC \\
tRF-24-Q99P9P9NF2 & 2.41 & 0.020747 & GCTTCTGTAGTGTAGTGGTTATCA & tRF-5 & ValCAC \\
tRF-30-6SXMSL73VL4Y & 2.19 & $1.13 \times 10^{-5}$ & GGCCGTGATCGTATAGTGGTTAGTACTCTG & tRF-5 & HisGTG \\
tRF-26-P4R8YP9LOND & 1.13 & 0.009501 & GCATGGGTGGTTCAGTGGTAGAATTC & tRF-5 & GlyGCC \\
tRF-30-XSXMSL73VL4Y & 1.48 & 0.016991 & TGCCGTGATCGTATAGTGGTTAGTACTCTG & tRF-5 & HisGTG
\end{tabular}

Database ID refers to the ID in MINTbase (https://cm.jefferson.edu/MINTbase/). tRF, transfer RNA-derived fragment; FC, fold change; FDR, false discovery rate.

tion were determined statistically using Pearson's Chi-square test. Measurement data are presented as the mean \pm standard deviation and were analyzed by paired Student's t-test for comparisons of two groups or one-way analysis of variance followed by Tukey's post hoc test for comparisons among multiple groups. $\mathrm{P}<0.05$ was considered to indicate a statistically significant difference.

\section{Results}

Associations between demographic and clinical characteristics of patients and differentiation of cancer. The demographic and clinical characteristics of the 30 patients are presented in Table I. Characteristics included sex, age and tumor size, location of hemicolon, TNM stage, lymph node metastasis and distant metastasis. There were no significant differences between the low and moderate/high differentiation groups.

Data mapping and results. After filtering with FastQC, the clean reads were mapped to different small RNA databases or sequences (Fig. 1A). Of all mapped reads from colon cancer tissues, $87.87 \%$ were miRNAs, $6.66 \%$ tRFs, $0.70 \%$ snRNAs, $0.58 \%$ snoRNAs and $0.01 \%$ known piRNAs (Fig. 1B). The various small RNAs in peritumor tissues were $85.91 \%$ miRNAs, $8.09 \%$ tRFs, $0.59 \%$ snRNAs, $0.46 \%$ snoRNAs and $0.01 \%$ piRNAs (Fig. 1B).

DE tRFs and miRNAs. As shown in Table III, a total of 13,838 tRFs, 2,588 miRNAs and 167 known piRNAs were identified in the six samples. The identified tRFs were of six types: 3'-half, 5'-half, i-tRF, tRF-1, tRF-3 and tRF-5 (Fig. 2A). In total, 16 tRFs (Table IV) and 26 miRNAs exhibited significant differential expression between the colon cancer and normal tissues with $\mid \log _{2}$ (fold change) $\mid>1$ and FDR $<0.05$ (Fig. 2B). No significantly DE known piRNAs were detected.

DE $m R N A$ and function analysis. From the GEPIA, 5,372 DE mRNAs were identified between colon adenocarcinoma and paired normal samples. Of these, 2,692 were significantly upregulated and 2,680 were significantly downregulated (Fig. 3A). The DE mRNAs were further investigated by $\mathrm{GO}$ and KEGG pathway enrichment analyses. The DE mRNAs were mainly enriched in cell proliferation-related GO terms, including extracellular matrix organization, cell adhesion, mitotic cell cycle, mitotic nuclear division, cell cycle, negative regulation of cell proliferation, G1/S transition of mitotic cell cycle, regulation of cell proliferation, positive regulation of cell proliferation, DNA replication and DNA strand elongation involved in DNA replication (Fig. 3B). In the KEGG pathway enrichment analysis, the DE mRNAs were primarily enriched in cell proliferation-related (including cell cycle, ECM-receptor interaction and DNA replication) and cancer-related (including p53 signaling replication, small cell lung cancer, pathways in cancer and PI3K-Akt signaling pathway) KEGG terms (Fig. 3C).

Integrated $t R F / m R N A / m i R N A$ analysis. Through literature mining, five of the $26 \mathrm{DE}$ miRNAs (hsa-miR-139-3p, hsa-miR-378a-3p, hsa-miR-424-3p, hsa-miR-483-3p and hsa-miR-615-3p) were selected as key miRNAs. The DE tRFs and five key miRNAs were common to and negatively correlated with 55 target genes. An integrated tRF-mRNA-miRNA 
A

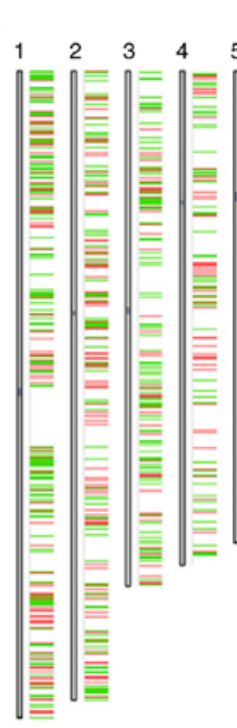

Differentially expressed genes on chromosomes
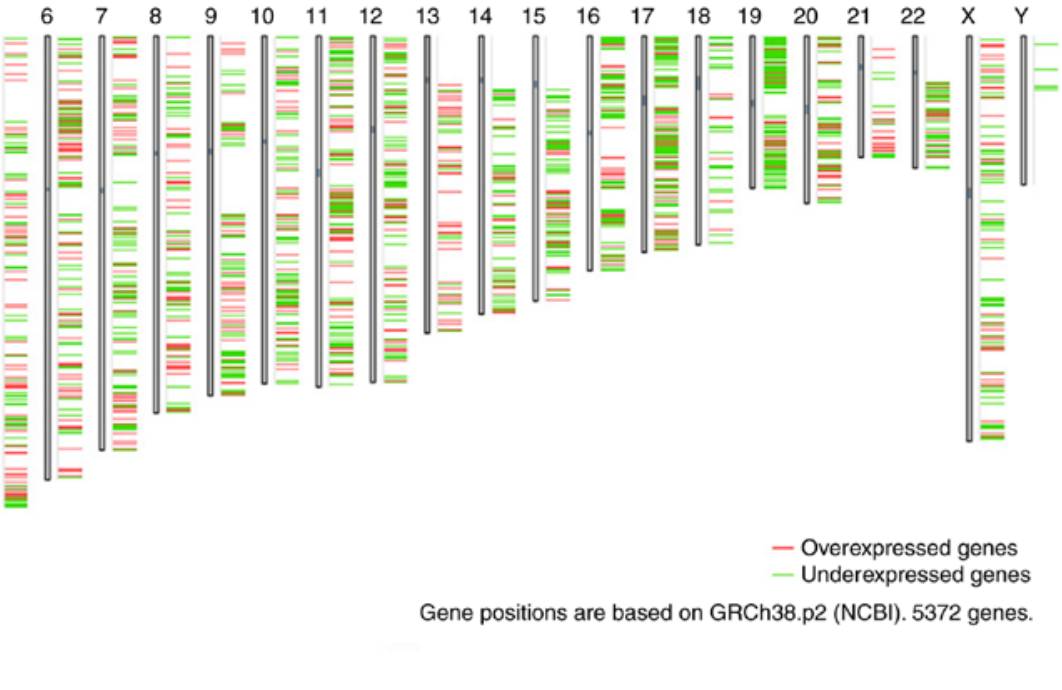

B

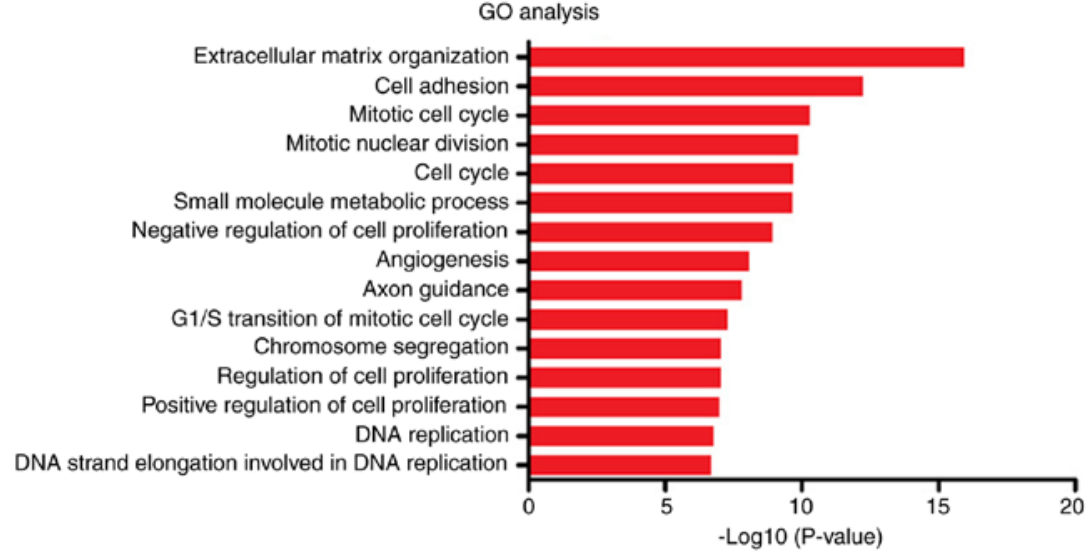

C

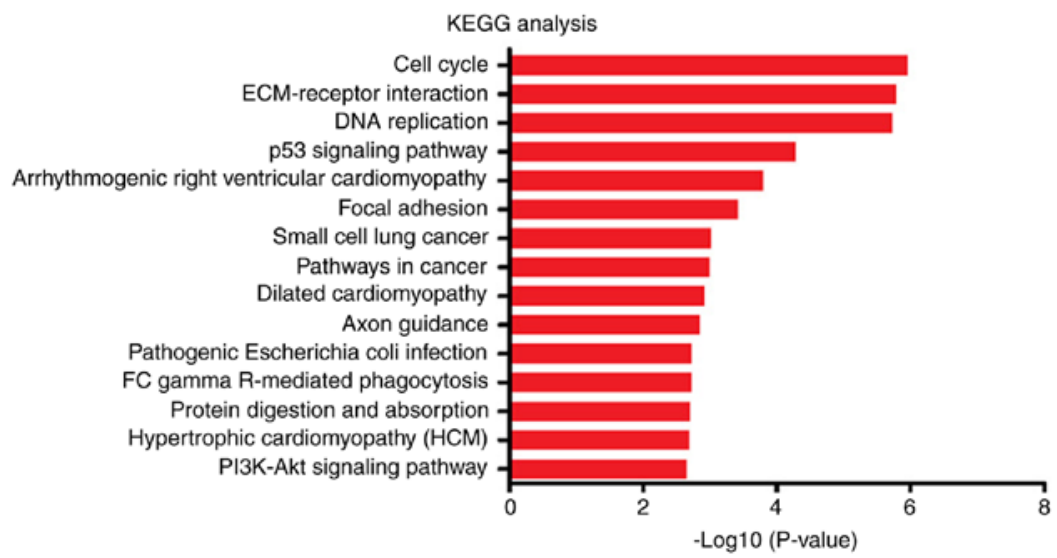

Figure 3. Analysis of DE mRNAs between colon cancer and paired normal tissues. (A) DE mRNAs encoded by chromosomal genes. (B) GO analysis of DE mRNAs. (C) Pathway analysis of DE mRNAs. DE, differentially expressed; GO, Gene Ontology; KEGG, Kyoto Encyclopedia of Genes and Genomes.

network was constructed (Fig. 4A). Next, the 55 potential targets of DE tRFs and key miRNAs were subjected to GO and pathway enrichment analyses. These potential targets were mainly enriched in the GO terms regulation of microtubule-based process, smooth muscle cell differentiation, heme biosynthetic process, water-soluble vitamin metabolic process and vitamin metabolic process (Fig. 4B). In the KEGG analyses, the potential targets were enriched in the terms cGMP-PKG signaling pathway, vitamin B6 metabolism, insulin secretion, ECM-receptor interaction and sulfur relay system (Fig. 4C).
The target genes MOCS1 and PDXK were enriched in the GO terms water-soluble vitamin metabolic process and vitamin metabolic process (Table V). PDXK was enriched in the pathway term vitamin B6 metabolism. KCNMA1, ADCY5 and NFATC4 were significantly enriched in the pathway term cGMP-PKG signaling pathway. From the integrated tRF-mRNA-miRNA network (Fig. 4A), tRF-26-P4R8YP9LOND was associated with MOCS1, tRF-24-NMEH623K25 and tRF-29-P27JPJ60MVJY were associated with $P D X K$, tRF-29-QU7BPN6ISBJO was associated with KCNMA1 and ADCY5, and tRF-27-Q99P9P9NH5N was associated with NFATC4. 
A
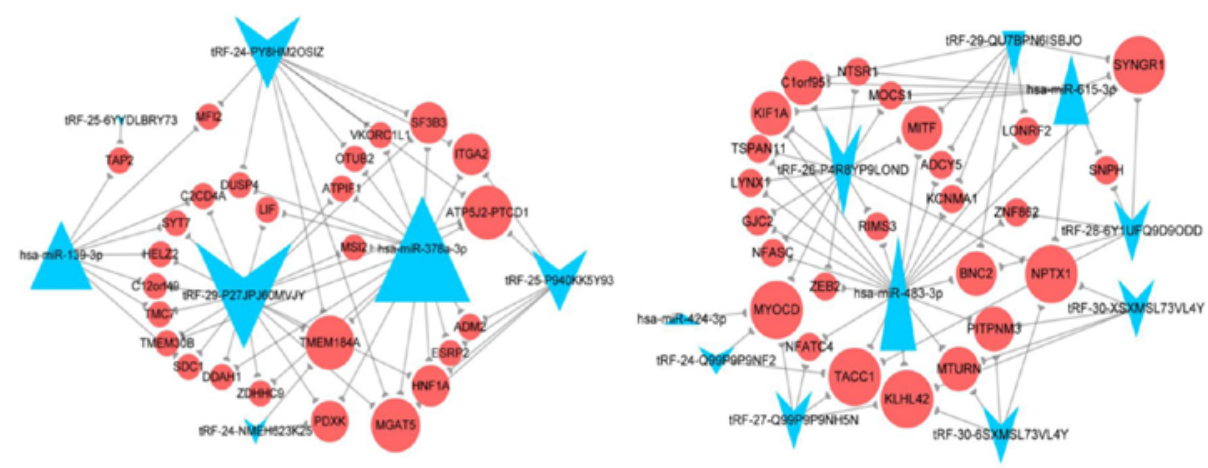

B
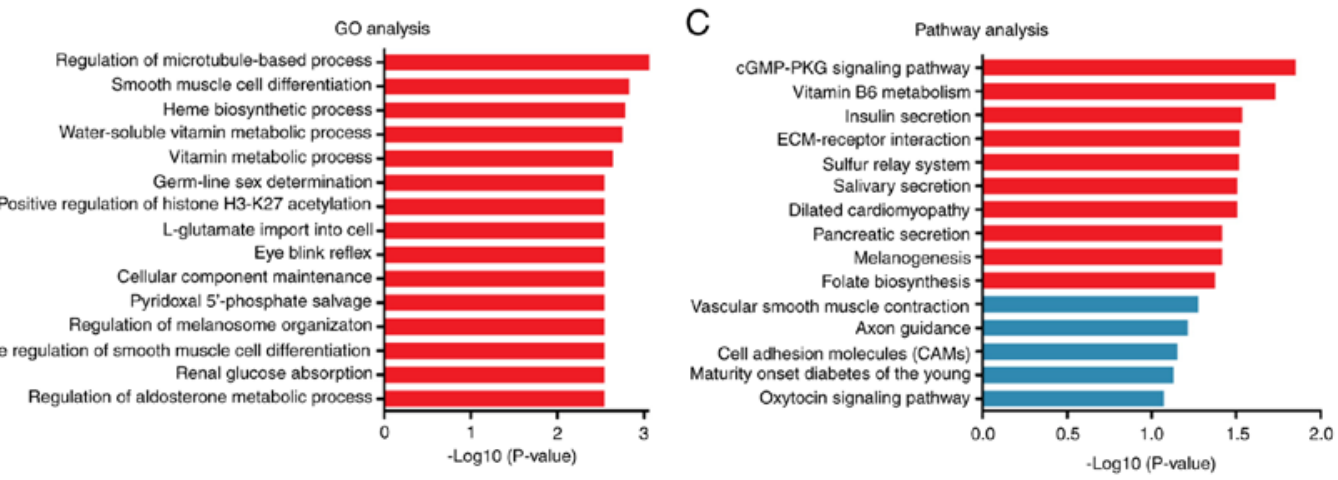

Figure 4. Integrated tRF/mRNA/miRNA analysis. (A) Potential targets that were common to and negative correlated with DE tRFs and key miRNAs. A network of downregulated miRNAs and tRFs and upregulated mRNAs is shown on the left. A network of upregulated miRNAs and tRFs and downregulated mRNAs is shown on the right. The size of the arrowheads, triangles and ovals represents the number of interacting targets. (B) GO and (C) pathway enrichment analyses of the potential targets. Arrowheads, triangles and ovals indicate DE tRFs, miRNAs and mRNAs, respectively. Blue and red indicate significantly downregulated and upregulated genes, respectively. miRNA, microRNA; tRF, transfer RNA-derived fragment; DE, differentially expressed; GO, Gene Ontology.
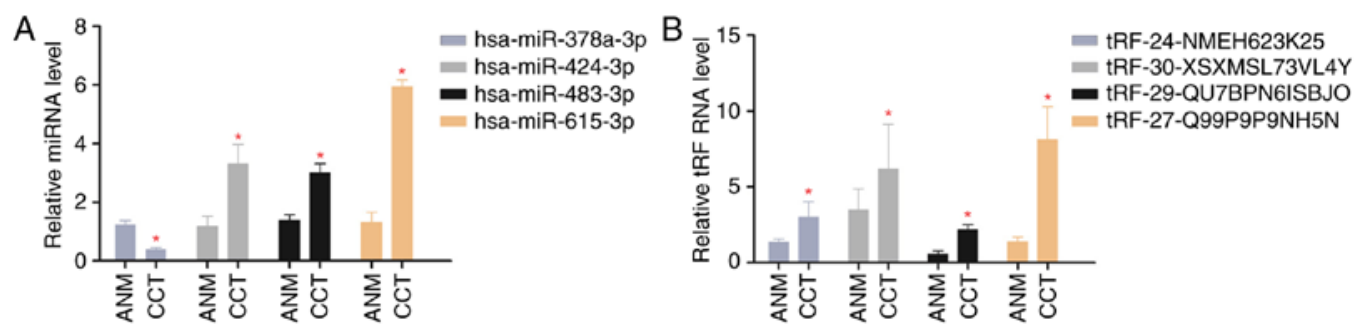

C

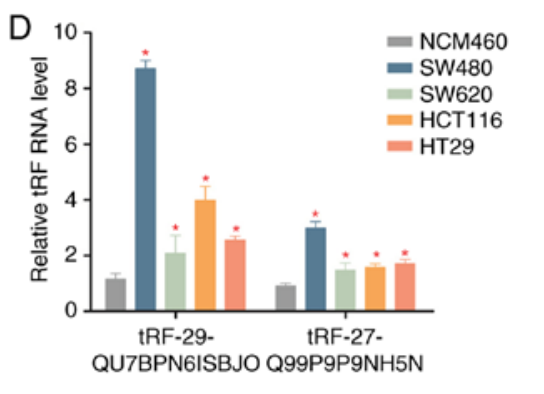

Figure 5. RT-qPCR analysis of key miRNA and candidate tRF expression. The differential expression of (A) four key miRNAs and (B) four tRFs was verified by RT-qPCR in CCT and ANM ( $\mathrm{n}=30)$. " $\mathrm{P}<0.05$ vs. ANM. (C) tRF expression in normal tissue and LD or M/HD tumor tissue. ${ }^{*} \mathrm{P}<0.05$ vs. N; " $\mathrm{P}<0.05$ vs. $\mathrm{LD}$. (D) tRF-29-QU7BPN6ISBJO and tRF-27-Q99P9P9NH5N expression levels in colon cancer cell lines were verified by RT-qPCR. "P<0.05 vs. NCM460. CCT, colon cancer tissue; ANM, adjacent normal mucosa; N, normal tissue; tRF, transfer RNA-derived fragment; miRNA, microRNA; LD, low differentiation; $\mathrm{M} / \mathrm{HD}$, moderate/high differentiation; RT-qPCR, reverse transcription-quantitative polymerase chain reaction.

Validation of key miRNAs and candidate tRF expression by $R T-q P C R$. Four key DE miRNAs, of which one was downregulated (hsa-miR-378a-3p) and three were upregulated (hsa-miR-424-3p, hsa-miR-483-3p, hsa-miR-615-3p) were verified by RT-qPCR in colon cancer tissue and adjacent normal mucosa ( $n=30$; Fig. 5A). Four DE tRFs
(tRF-24-NMEH623K 25, tRF-30-XSXMSL73VL4Y, tRF-29-QU7BPN6ISBJO and tRF-27-Q99P9P9NH5N) were significantly upregulated in colon cancer tissue compared with adjacent normal mucosa (Fig. 5B). Notably, DE tRF expression increased with tumor differentiation (Fig. 5C). Furthermore, tRF-29-QU7BPN6ISBJO and tRF-27-Q99P9P9NH5N were 
Table V. GO and pathway terms and enriched genes.

\begin{tabular}{lllll}
\hline ID & \multicolumn{1}{c}{ Term } & \multicolumn{1}{c}{ Genes } & P-value & Enrichment \\
\hline GO:0032886 & Regulation of microtubule-based process & TACC1, KLHL42 & 0.0005298 & 57.690476 \\
GO:0051145 & Smooth muscle cell differentiation & MYOCD, NFATC4 & 0.0015027 & 34.614286 \\
GO:0006783 & Heme biosynthetic process & ATPIF1, HNF1A & 0.0016579 & 32.965986 \\
GO:0006767 & Water-soluble vitamin metabolic process & MOCS1, GSTO2, PDXK & 0.00177 & 12.511188 \\
GO:0006766 & Vitamin metabolic process & MOCS1, GSTO2, PDXK & 0.0023028 & 11.411303 \\
GO:0018992 & Germ-line sex determination & TMEM184A & 0.002889 & 346.14286 \\
GO:1901676 & Positive regulation of histone H3-K27 & LIF & 0.002889 & 346.14286 \\
& acetylation & & & \\
GO:1990123 & L-glutamate import into cell & NTSR1 & 0.002889 & 346.14286 \\
GO:0060082 & Eye blink reflex & KCNMA1 & 0.002889 & 346.14286 \\
GO:0043954 & Cellular component maintenance & MYOCD & 0.002889 & 346.14286 \\
GO:0009443 & Pyridoxal 5'-phosphate salvage & PDXK & 0.002889 & 346.14286 \\
GO:1903056 & Regulation of melanosome organization & ZEB2 & 0.002889 & 346.14286 \\
GO:2000724 & Positive regulation of cardiac vascular & MYOCD & 0.002889 & 346.14286 \\
& smooth muscle cell differentiation & & & \\
GO:0035623 & Renal glucose absorption & HNF1A & 0.002889 & 346.14286 \\
GO:0032344 & Regulation of aldosterone metabolic process & KCNMA1 & 0.002889 & 346.14286 \\
PATH:04022 & cGMP-PKG signaling pathway & KCNMA1, ADCY5, & 0.0140781 & 5.8015398 \\
& & NFATC4 & & \\
PATH:00750 & Vitamin B6 metabolism & PDXK & 0.0184421 & 53.825397 \\
PATH:04911 & Insulin secretion & KCNMA1, ADCY5 & 0.0291694 & 7.4241927 \\
PATH:04512 & ECM-receptor interaction & ITGA11, SDC1 & 0.0304212 & 7.2573569 \\
PATH:04122 & Sulfur relay system & MOCS1, KCNMA1, & 0.0305564 & 32.295238 \\
PATH:05414 & Dilated cardiomyopathy & ADCY5 & & \\
PATH:04972 & Pancreatic secretion & ITGA11, ADCY5 & 0.0310552 & 7.1767196 \\
PATH:04916 & Melanogenesis & KCNMA1, ADCY5 & 0.0383707 & 6.3950967 \\
PATH:00790 & Folate biosynthesis & MITF, ADCY5 & 0.0383707 & 6.3950967 \\
& MOCS1 & 0.0425282 & 23.068027 \\
\hline
\end{tabular}

IDs beginning with GO and PATH indicate GO terms and pathway terms, respectively. GO, Gene Ontology.

overexpressed in colon cancer cell lines (SW480, SW620, HCT116 and HT29) compared with a colon epithelial cell line (NCM460), as verified by RT-qPCR (Fig. 5D).

\section{Discussion}

In the present study, 16 DE tRFs and 26 DE miRNAs were identified between colon cancer and normal peritumor tissues by small RNA sequencing. In addition, 5,327 DE mRNAs between colon cancer and normal tissues were screened by GEPIA. In the GO and pathway analyses, the DE mRNAs were enriched in cell proliferation-related GO and pathway terms. Recent evidence suggests that tRFs exert their effects in a similar way to miRNAs (27). Therefore the subsequent analysis and experiments were based on the hypothesis that tRFs and miRNA target the same DE mRNAs and perform similar biological functions in the development of colon cancer.

Among the 26 DE miRNAs between colon cancer and paired normal tissues, five key miRNAs (hsa-miR-139-3p, hsa-miR-378a-3p, hsa-miR-424-3p, hsa-miR-483-3p and hsa-miR-615-3p) have been implicated in colon cancer. For example, low expression of miR-139a-3p and miR-378a-3p is associated with poor survival of colorectal cancer patients (28-30). Overexpression of miR-378a-3p suppresses colon cancer cell proliferation and induces apoptosis (30). miR-424-3p is upregulated in primary and metastatic cancers and colorectal cancer cell lines, and its overexpression is associated with poor outcomes in patients with colorectal cancer (31). miR-483-3p was identified to be significantly upregulated in tissue and blood samples of colorectal cancer patients, and to promote the proliferation of colorectal cancer cells and inhibit DLC-1 expression by targeting its 3'UTR. Furthermore, transfection with an miR-483-3p antagomir suppressed the growth of colorectal cancer cells $(32,33)$. High expression of miR-615-3p was reported in right-sided colon cancer (34). In the present study, hsa-miR-139-3p and hsa-miR-378a-3p were identified to be downregulated in colon cancer tissues, whereas hsa-miR-424-3p, hsa-miR-483-3p and hsa-miR-615-3p were identified to be upregulated. These results are consistent with previous reports. Thus, these key miRNAs were selected to analyze the roles of tRFs in colon cancer. 
To explore the roles of tRFs in colon cancer, an integrated $\mathrm{tRF} / \mathrm{mRNA} / \mathrm{miRNA}$ analysis was performed. The mRNAs in the constructed network were enriched in the GO terms regulation of microtubule-based process, smooth muscle cell differentiation, heme biosynthetic process, water-soluble vitamin metabolic process and vitamin metabolic process. The top five pathway terms were cGMP-PKG signaling pathway, vitamin B6 metabolism, insulin secretion, ECM-receptor interaction and sulfur relay system. Several studies have reported that vitamins play important roles in colon cancer. Dietary vitamin B6 and D inhibited oncogenesis of colon cancer in a murine model and human colon cancer cells (35-37). Low intake of folate and vitamin B6, along with p53 overexpression, increased the risk of colon cancer development (38). Increased vitamin B6 altered expression of genes in the colon and inhibited the development and progression of colon cancer by suppressing cancer cell proliferation $(39,40)$. Thus, vitamin B6 plays an important role in colon cancer by activating p53 and increasing the expression of p21 (41). Vitamin K1 treatment inhibited the proliferation and induced apoptosis of human colon cancer cells (42). These reports suggest that vitamin metabolic processes are intensively associated with colon tumorigenesis. In the present study, the target genes of tRF-26-P4R8YP9LOND, tR F-2 5-P940KK 5 Y93, tRF-30-XSXMSL73 V L 4Y, tRF-24-NMEH623K25 and tRF-29-P27JPJ60MVJY were associated with vitamin metabolism-related processes and pathways (water-soluble vitamin metabolic process, vitamin metabolic process and vitamin B6 metabolism). In summary, these findings indicate that tRFs, particularly tRF-26-P4R8YP9LOND, tRF-25-P940KK5Y93, tRF-30-XSXMSL73VL4Y, tRF-24-NMEH623K25 and tRF-29-P27JPJ60MVJY, may regulate vitamin levels and contribute to the development and progression of colon cancer.

In addition, the cGMP-PKG signaling pathway can be activated by the non-steroidal anti-inflammatory drug sulindac sulfide, which is known to inhibit tumorigenesis by blocking the proliferation and inducing apoptosis of colon cancer cells $(43,44)$. In the present study, the target genes of tRF-29-QU7BPN6ISBJO and tRF-27-Q99P9P9NH5N were identified to be enriched in the pathway term cGMP-PKG signaling pathway. This result suggests that tRF-29-QU7BPN6ISBJO and tRF-27-Q99P9P9NH5N may be involved in the development of colon cancer via the cGMP-PKG signaling pathway.

In conclusion, the present study identified several tRFs that may be involved in the development and progression of colon cancer via vitamin metabolism-related processes and pathways and the cGMP-PKG signaling pathway. However, the study has certain limitations that must be considered. First, only three samples were studied. Second, the expression pattern of the identified DE tRFs and their association with the identified target genes require further verification. The interaction between tRFs and their target genes could be detected using the AGO2-RIP method and a dual-luciferase reporter gene system. Finally, the specific role of tRFs in colon cancer should be analyzed further. tRF expression should be evaluated following exogenous expression or interference of target genes, and the effect of tRFs and their target mRNAs on the occurrence and development of tumor cells should be verified through cell function experiments, including cell proliferation, migration and invasion assays.

\section{Acknowledgements}

Not applicable.

\section{Funding}

The present study was supported by the National Natural Science Foundation of China (grant no. 81301825) and the Training Program for Medical Reserve Talents of the Health and Family Planning Commission of Yunnan Province (grant nos. H-201641 and H-201624).

\section{Availability of data and materials}

The datasets used in the present study are available from the corresponding author upon reasonable request.

\section{Authors' contributions}

YuL, JQ and WX (first author) conceived and design the study. WX (first author), XW, XC, WX (fourth author), YaL, CL and QL performed the experiments. WX (first author) wrote the manuscript. XW, JQ and YuL reviewed and edited the manuscript. All authors read and approved the manuscript and agree to be accountable for all aspects of the research and for ensuring that the accuracy or integrity of all parts of this work are appropriately investigated and resolved.

\section{Ethics approval and consent to participate}

All experimental protocols were approved by the Human Ethics Committee of Kunming Medical University (Kunming, China). Written informed consent was obtained from all patients.

\section{Patient consent for publication}

Not applicable.

\section{Competing interests}

The authors declare that they have no competing interests.

\section{References}

1. Ferlay J, Soerjomataram I, Dikshit R, Eser S, Mathers C, Rebelo M, Parkin DM, Forman D and Bray F: Cancer incidence and mortality worldwide: Sources, methods and major patterns in GLOBOCAN 2012. Int J Cancer 136: E359-E386, 2015.

2. Jaganathan SK, Vellayappan MV, Narasimhan G and Supriyanto E: Role of pomegranate and citrus fruit juices in colon cancer prevention. World J Gastroenterol 20: 4618-4625, 2014.

3. Chen J, Elfiky A, Han M, Chen C and Saif MW: The role of Src in colon cancer and its therapeutic implications. Clin Colorectal Cancer 13: 5-13, 2014.

4. Kawaji H, Nakamura M, Takahashi Y, Sandelin A, Katayama S, Fukuda S, Daub CO, Kai C, Kawai J, Yasuda J, et al: Hidden layers of human small RNAs. BMC Genomics 9: 157, 2008.

5. Cole C, Sobala A, Lu C, Thatcher SR, Bowman A, Brown JW, Green PJ,Barton GJ and Hutvagner G: Filtering of deep sequencing data reveals the existence of abundant Dicer-dependent small RNAs derived from tRNAs. RNA 15: 2147-2160, 2009. 
6. Lee YS, Shibata Y, Malhotra A and Dutta A: A novel class of small RNAs: TRNA-derived RNA fragments (tRFs). Genes Dev 23: 2639-2649, 2009

7. Thompson DM and Parker R: Stressing out over tRNA cleavage. Cell 138: 215-219, 2009.

8. Martens-Uzunova ES, Olvedy $\mathrm{M}$ and Jenster G: Beyond microRNA - novel RNAs derived from small non-coding RNA and their implication in cancer. Cancer Lett 340: 201-211, 2013.

9. Kumar P, Anaya J, Mudunuri SB and Dutta A: Meta-analysis of tRNA derived RNA fragments reveals that they are evolutionarily conserved and associate with AGO proteins to recognize specific RNA targets. BMC Biol 12: 78, 2014.

10. Calin GA and Croce CM: MicroRNA signatures in human cancers. Nat Rev Cancer 6: 857-866, 2006.

11. Muller S, Raulefs S, Bruns P, Afonso-Grunz F, Plötner A Thermann R, Jäger C, Schlitter AM, Kong B, Regel I, et al: Next-generation sequencing reveals novel differentially regulated mRNAs, IncRNAs, miRNAs, sdRNAs and a piRNA in pancreatic cancer. Mol Cancer 14: 94, 2015.

12. Olvedy M, Scaravilli M, Hoogstrate Y, Visakorpi T, Jenster G and Martens-Uzunova ES: A comprehensive repertoire of tRNA-derived fragments in prostate cancer. Oncotarget 7: 24766-24777, 2016.

13. Balatti V, Nigita G, Veneziano D, Drusco A, Stein GS Messier TL, Farina NH, Lian JB, Tomasello L, Liu CG, et al: tsRNA signatures in cancer. Proc Natl Acad Sci U S A 114 8071-8076, 2017

14. Maute RL, Schneider C, Sumazin P, Holmes A, Califano A Basso K and Dalla-Favera R: tRNA-derived microRNA modulates proliferation and the DNA damage response and is down-regulated in B cell lymphoma. Proc Natl Acad Sci U S A 110: 1404-1409, 2013

15. Kozomara A and Griffiths-Jones S: miRBase: Annotating high confidence microRNAs using deep sequencing data. Nucleic Acids Res 42: D68-D73, 2014.

16. Balatti V, Rizzotto L, Miller C, Palamarchuk A, Fadda P, Pandolfo R, Rassenti LZ, Hertlein E, Ruppert AS, Lozanski A, et al: TCL1 targeting $m i R-3676$ is codeleted with tumor protein p53 in chronic lymphocytic leukemia. Proc Natl Acad Sci USA 112: 2169-2174, 2015 .

17. Qi L and Ding Y: Screening of differentiation-specific molecular biomarkers for colon cancer. Cell Physiol Biochem 46: 2543-2550, 2018.

18. Yang L, Wang S, Zhou Y, Lai S, Xiao G, Gazdar A and Xie Y: Evaluation of the 7th and 8th editions of the AJCC/UICC TNM staging systems for lung cancer in a large North American cohort. Oncotarget 8: 66784-66795, 2017.

19. Nawrocki EP, Burge SW, Bateman A, Daub J, Eberhardt RY, Eddy SR, Floden EW, Gardner PP, Jones TA, Tate J, et al: Rfam 12.0: Updates to the RNA families database. Nucleic Acids Res 43: D130-D137, 2015

20. Kalvari I, Argasinska J, Quinones-Olvera N, Nawrocki EP, Rivas E, Eddy SR, Bateman A, Finn RD and Petrov AI: Rfam 13.0: Shifting to a genome-centric resource for non-coding RNA families. Nucleic Acids Res 46: D335-D342, 2018

21. Tang Z, Li C, Kang B, Gao G and Zhang Z: GEPIA: A web server for cancer and normal gene expression profiling and interactive analyses. Nucleic Acids Res 45: W98-W102, 2017.

22. Shannon P, Markiel A, Ozier O, Baliga NS, Wang JT, Ramage D, Amin N, Schwikowski B and Ideker T: Cytoscape: A software environment for integrated models of biomolecular interaction networks. Genome Res 13: 2498-2504, 2003

23. Kanehisa M and Goto S: KEGG: Kyoto encyclopedia of genes and genomes. Nucleic Acids Res 28: 27-30, 2000.

24. Hulsegge I, Kommadath A and Smits MA: Globaltest and GOEAST: Two different approaches for gene ontology analysis. BMC Proc 4 (Suppl 3): S10, 2009.

25. Ashburner M, Ball CA, Blake JA, Botstein D, Butler $\mathrm{H}$, Cherry JM, Davis AP, Dolinski K, Dwight SS, Eppig JT, et al: Gene ontology: Tool for the unification of biology. The Gene Ontology Consortium. Nat Genet 25: 25-29, 2000.
26. Livak KJ and Schmittgen TD: Analysis of relative gene expression data using real-time quantitative PCR and the $2^{-\Delta \Delta C_{\mathrm{T}}}$ method. Methods 25: 402-408, 2001.

27. Kuscu C, Kumar P, Kiran M, Su Z, Malik A and Dutta A: tRNA fragments (tRFs) guide Ago to regulate gene expression post-transcriptionally in a Dicer independent manner. RNA 24 : 1093-1105, 2018.

28. Liu X, Duan B, Dong Y, He C, Zhou H, Sheng H, Gao H and Zhang X: MicroRNA-139-3p indicates a poor prognosis of colon cancer. Int J Clin Exp Pathol 7: 8046-8052, 2014.

29. Ng L, Wan TM, Man JH, Chow AK, Iyer D, Chen G, Yau TC, Lo OS, Foo DC, Poon JT, et al: Identification of serum miR-139-3p as a non-invasive biomarker for colorectal cancer. Oncotarget 8: 27393-27400, 2017.

30. Li H, Dai S, Zhen T, Shi H, Zhang F, Yang Y, Kang L, Liang Y and Han A: Clinical and biological significance of miR-378a-3p and miR-378a-5p in colorectal cancer. Eur J Cancer 50: 1207-1221, 2014.

31. Torres S, Garcia-Palmero I, Bartolome RA, FernandezAceñero MJ, Molina E, Calviño E, Segura MF and Casal JI: Combined miRNA profiling and proteomics demonstrates that different miRNAs target a common set of proteins to promote colorectal cancer metastasis. J Pathol 242: 39-51, 2017.

32. Yong FL, Law CW and Wang CW: Potentiality of a triple microRNA classifier: MiR-193a-3p, miR-23a and miR-338-5p for early detection of colorectal cancer. BMC cancer 13: 280, 2013.

33. Cui H, Liu Y, Jiang J, Liu Y, Yang Z, Wu S, Cao W, Cui IH and Yu C: IGF2-derived miR-483 mediated oncofunction by suppressing DLC-1 and associated with colorectal cancer. Oncotarget 7: 48456-48466, 2016.

34. Schee K, Lorenz S, Worren MM, Günther CC, Holden M, Hovig E, Fodstad O, Meza-Zepeda LA and Flatmark K: Deep sequencing the MicroRNA transcriptome in colorectal Cancer. PloS One 8: e66165, 2013.

35. Komatsu S, Yanaka N, Matsubara K and Kato N: Antitumor effect of vitamin B6 and its mechanisms. Biochim Biophys Acta 1647: 127-130, 2003

36. Meeker S, Seamons A, Paik J, Treuting PM, Brabb T, Grady WM and Maggio-Price L: Increased dietary vitamin D suppresses MAPK signaling, colitis, and colon cancer. Cancer Res 74: 4398-4408, 2014

37. Pereira F, Larriba MJ and Munoz A: Vitamin D and colon cancer. Endocr Relat Cancer 19: R51-R71, 2012.

38. Schernhammer ES, Ogino $S$ and Fuchs CS: Folate and vitamin $B_{6}$ intake and risk of colon cancer in relation to 553 expression. Gastroenterology 135: 770-780, 2008

39. Toya K, Hirata A, Ohata T, Sanada Y, Kato N and Yanaka N: Regulation of colon gene expression by vitamin B6 supplementation. Mol Nutr Food Res 56: 641-652, 2012.

40. Komatsu SI, Watanabe H, Oka T, Tsuge H, Nii H and Kato N: Vitamin B-6-supplemented diets compared with a low vitamin B-6 diet suppress azoxymethane-induced colon tumorigenesis in mice by reducing cell proliferation. J Nutr 131: 2204-2207, 2001

41. Zhang P, Suidasari S, Hasegawa T, Yanaka N and Kato N: Vitamin $\mathrm{B}_{6}$ activates p53 and elevates p21 gene expression in cancer cells and the mouse colon. Oncol Rep 31: 2371-2376, 2014.

42. Orlando A, Linsalata M, Tutino V, D'Attoma B, Notarnicola M and Russo F: Vitamin K1 exerts antiproliferative effects and induces apoptosis in three differently graded human colon cancer cell lines. Biomed Res Int 2015: 296721, 2015.

43. Li N, Xi Y, Tinsley HN, Gurpinar E, Gary BD, Zhu B, Li Y, Chen X, Keeton AB, Abadi AH, et al: Sulindac selectively inhibits colon tumor cell growth by activating the cGMP/PKG pathway to suppress Wnt/ $\beta$-catenin signaling. Mol Cancer Ther 12: 1848-1859, 2013.

44. Li N, Chen X, Zhu B, Ramírez-Alcántara V, Canzoneri JC, Lee K, Sigler S, Gary B, Li Y, Zhang W, et al: Suppression of $\beta$-catenin/TCF transcriptional activity and colon tumor cell growth by dual inhibition of PDE5 and 10 . Oncotarget 6 : 27403-27415, 2015. 